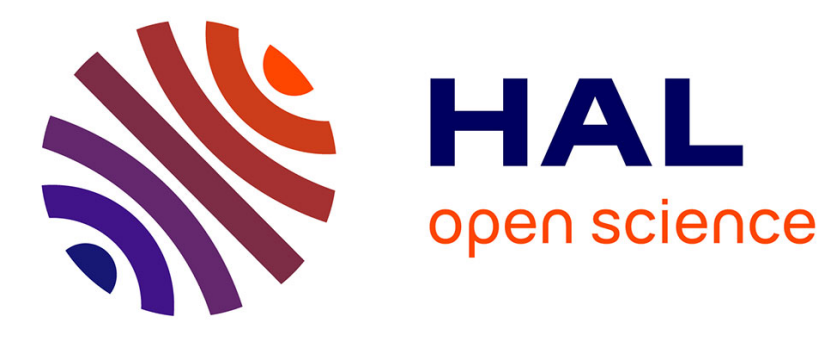

\title{
Thick inductive cross shaped metal meshes
}

Karl Dieter Möller, Oren Sternberg, Haim Grebel, Philippe Lalanne

\section{To cite this version:}

Karl Dieter Möller, Oren Sternberg, Haim Grebel, Philippe Lalanne. Thick inductive cross shaped metal meshes. Journal of Applied Physics, 2002, 91 (12), pp.9461-9465. 10.1063/1.1476073 . hal00878109

\section{HAL Id: hal-00878109 \\ https://hal-iogs.archives-ouvertes.fr/hal-00878109}

Submitted on 29 Oct 2013

HAL is a multi-disciplinary open access archive for the deposit and dissemination of scientific research documents, whether they are published or not. The documents may come from teaching and research institutions in France or abroad, or from public or private research centers.
L'archive ouverte pluridisciplinaire $\mathbf{H A L}$, est destinée au dépôt et à la diffusion de documents scientifiques de niveau recherche, publiés ou non, émanant des établissements d'enseignement et de recherche français ou étrangers, des laboratoires publics ou privés. 


\title{
Thick inductive cross shaped metal meshes
}

\author{
K. D. Möller ${ }^{\mathrm{a})}$ and O. Sternberg \\ Department of Physics and Electronic Imaging Center, New Jersey Institute of Technology, Newark, \\ New Jersey 07102 \\ H. Grebel \\ Electronic Imaging Center and Department of Electrical Engineering, New Jersey Institute of Technology, \\ Newark, New Jersey 07102 \\ Philippe Lalanne \\ Laboratoire Charles Fabry de l'Institut de'Optique, Center National de la Recherche Scientifique, \\ BP 147, F-91403 Orsey Cedex, France
}

(Received 14 December 2001; accepted for publication 13 March 2002)

\begin{abstract}
Experimental data for inductive cross shaped metal meshes with periodicity constant $g=20 \mu \mathrm{m}$ have been reproduced by the Micro-Stripes simulation program for various thicknesses. A similar calculation has been performed with the Fourier modal method for cross shaped meshes with periodicity constant $g=1 \mu \mathrm{m}$. The transmittances of all these meshes show similar resonance peaks and the same dependence on thickness. A rudimentary coupled oscillator model describes very well the dependence on thickness of the peaks, suggesting that the transmittance of all metal meshes in the short and long wavelength region are excited surface waves coupled by waveguide modes of the openings. (C) 2002 American Institute of Physics. [DOI: 10.1063/1.1476073]
\end{abstract}

\section{INTRODUCTION}

Recent experimental and theoretical work ${ }^{1-3}$ on metallic membranes perforated by subwavelength apertures has attracted much interest because of potential applications for filtering and confinement of light in the visible spectral region. This recent work shows many similarities to work done on inductive metal meshes used as filters in the long wavelength region. We will show that for meshes with cross shaped openings there is similar appearance, in both wavelength regions, of transmittance peaks with respect to the dependence on thickness of the meshes. The transmittances, normalized by considering the ratio of area of the aperture to the area of the unit cell, are also about the same. Electroformed metal meshes have been used since the 1960s as Fabry-Perot reflectors in the long wavelength region. ${ }^{4}$ These square shaped metal meshes, called by Ulrich" "inductive" grids, had thicknesses of less than $10 \%$ of the periodicity constant and are called thin meshes. Ulrich introduced metal meshes with cross shaped patterns and Chase and Joseph studied the transmittance dependence on the shape of the cross. ${ }^{6}$ In the short wavelength region, Byrne et al. ${ }^{7}$ investigated inductive cross shaped metal meshes on a substrate. All these meshes have a broad resonance transmittance at wavelength slightly longer than the periodicity constant, with peak wavelengths depending sensitively on the geometrical parameters of the cross.

Thick metal meshes with thickness larger than $10 \%$ of the periodicity constant have been produced by Ruprecht et $a l .{ }^{8}$ The meshes have been manufactured for a periodicity constant of $g=20 \mu \mathrm{m}$ using the LIGA method (a lithographic-electroforming process). The transmittances of

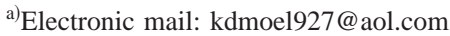

these free standing cross shaped metal meshes show an additional peak at shorter wavelength, strongly depending on the thickness of the mesh. Compton et al. ${ }^{9}$ have predicted these peaks for thick free standing cross shaped metal meshes and Möller et al. ${ }^{10}$ have given an interpretation of Ruprecht et al.'s work. In the short wavelength region, Ebbesen et al. ${ }^{1}$ and Grupp et al. ${ }^{2}$ have presented experimental studies of metal meshes with round holes and MartinMoreno et $a l .^{3}$ have calculated similar meshes with square shaped openings. In this article we show that all these metal meshes in the short and long wavelength region have similar transmittances with identical peak dependence on the thickness. Additionally, we present a rudimental model, expressed in terms of transmission line theory that describes very well all resonance peaks of the mesh.

\section{THICK CROSS SHAPED INDUCTIVE METAL MESHES}

\section{A. Experimental data and simulations \\ 1. Cross shaped meshes with periodicity constant $g=20 \mu m$}

a. Simulations. We have used the Micro-Stripes simulation program $^{11}$ for the calculation of the transmittance of cross shaped metal meshes, assuming a surface impedance of $1.635 \Omega \mathrm{cm}$ and geometrical parameters of the crosses of $g$ $=20 \mu \mathrm{m}, 2 a=1.5 \mu \mathrm{m}$, and $2 b=3 \mu \mathrm{m}$ (see Fig. 1). In Figs. 2(a)-2(c) the transmittance is shown for thicknesses of 0.2 , $3.2,6.4,9.6,12.8,16,19.2,22.4$, and $25.6 \mu \mathrm{m}$. In the longer wavelength region there are peaks shifting only slightly to shorter wavelengths, we call them "resonance peaks." At shorter wavelengths there are peaks which are largely dependent on the thickness of the mesh; we call them "thickness peaks." The thickness peaks appear first at a wavelength close to the periodicity constant $g$ and shift with increasing 

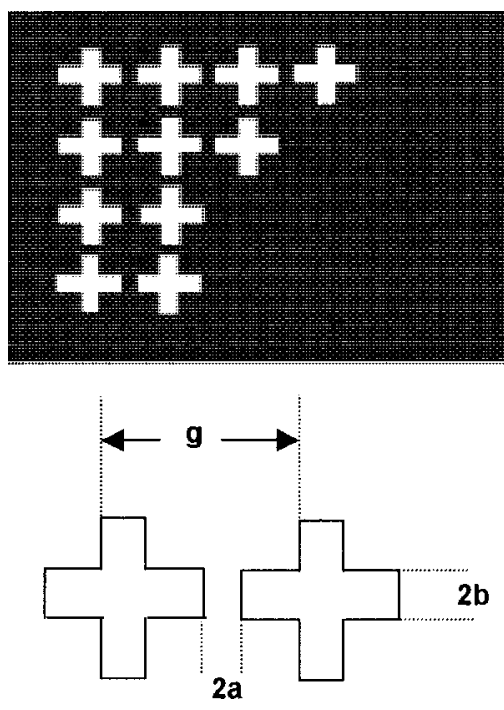

FIG. 1. Inductive cross shaped mesh and geometrical parameters (black is metal). Periodicity constant $g$, separation of crosses $2 a$, width of cross arms $2 b$, and metal thickness of the mesh $t$.

thickness towards the resonance peak. After a certain thickness of the mesh, a new series of thickness peaks appears with its first peak having again a wavelength close to the periodicity constant.

We have plotted in Fig. 3 all peak wavelengths divided by the periodicity constant $(\lambda / g)$ depending on the thickness divided by the periodicity constant $(t / g)$ for the range $0-1.5$.

b. Experiments. Ruprecht et al. ${ }^{8}$ have manufactured free standing metal meshes of excellent quality with the same geometrical parameters as used above of $g=20 \mu \mathrm{m}, 2 a$ $=1.5 \mu \mathrm{m}$, and $2 b=3 \mu \mathrm{m}$ and for thicknesses of 11,20 , and $29 \mu \mathrm{m}$. The transmittance is also shown in Fig. 3 and there is excellent agreement of the experimental data and simulations (within a few percent).

\section{Cross shaped meshes with periodicity constant $g=1 \mu m$}

In the short wavelength region we have used the Fourier modal method ${ }^{12}$ to calculate the transmittance of free standing cross shaped metal meshes with geometrical parameters of $g=1 \mu \mathrm{m}, 2 a=0.2 \mu \mathrm{m}$, and $2 b=0.11 \mu \mathrm{m}$ for thicknesses of $0.2,0.4,0.8,1,1.6$, and $2 \mu \mathrm{m}$ [see Figs. 4(a) and 4(b)]. We call the longer wavelength peaks "resonance peaks" and the shorter wavelength peaks "thickness peaks." The appearance depending on thickness is very similar to that found for the case of $g=20 \mu \mathrm{m}$. In Fig. 5 we have plotted for the range of $0-2$ the peak wavelength divided by the periodicity constant $(\lambda / g)$ depending on the thickness divided by the periodicity constant $(t / g)$.

Such calculations have been repeated for cross shaped metal meshes with similar ratios of $a / g$ and $b / g$ for periodicity constants $g=2.14$ and $3 \mu \mathrm{m}$. The results of the dependence on thickness of resonance and thickness peaks are the same as shown in Fig. 5.

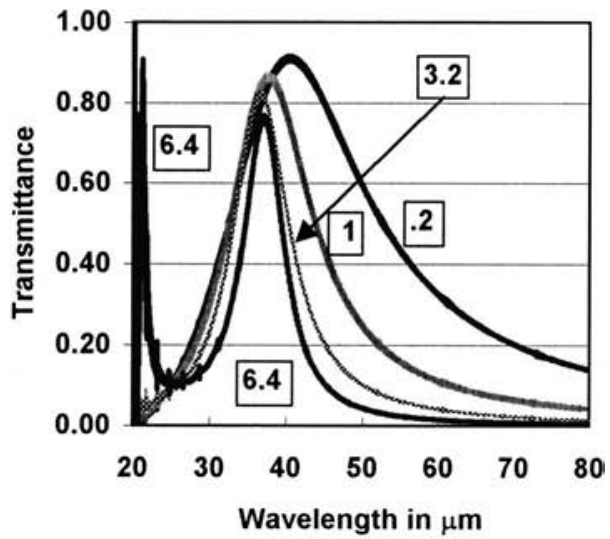

(a)

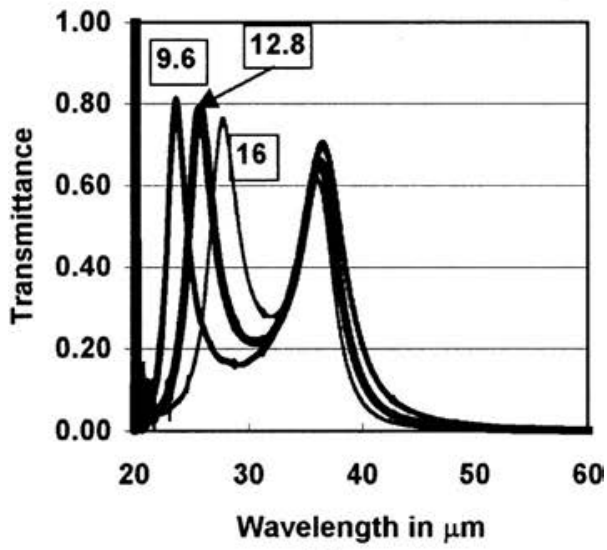

(b)

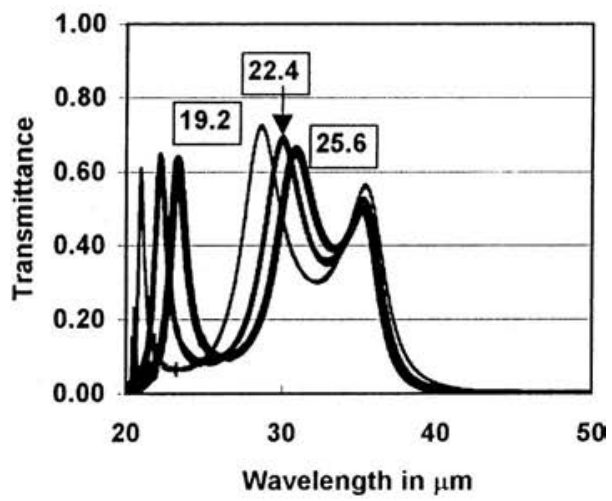

(c)

FIG. 2. (a) Transmittance of inductive cross shaped meshes calculated with the Micro-Stripes program. Geometrical parameters $g=20 \mu \mathrm{m}, \quad 2 a$ $=1.5 \mu \mathrm{m}$, and $2 b=3 \mu \mathrm{m}$. The metal thickness $0.2,1,3.2$, and $6.4 \mu \mathrm{m}$ are indicated in the graph area. The resonance peaks shift to shorter wavelength with increasing metal thicknesses (see also Fig. 3). (b) Transmittance of inductive cross shaped meshes calculated with the Micro-Stripes program. Geometrical parameters $g=20 \mu \mathrm{m}, 2 a=1.5 \mu \mathrm{m}$, and $2 b=3 \mu \mathrm{m}$. The metal thickness 9.6, 12.8, and $16 \mu \mathrm{m}$ are indicated in the graph area. The long wavelength peaks are resonance peaks, the short wavelength peaks are the first series of thickness peaks (see also Fig. 3). (c) Transmittance of inductive cross shaped meshes calculated with the Micro-Stripes program. Geometrical parameters $g=20 \mu \mathrm{m}, 2 a=1.5 \mu \mathrm{m}$, and $2 b=3 \mu \mathrm{m}$. The metal thickness $19.2,22.4$, and $25.6 \mu \mathrm{m}$ are indicated in the graph area. The longest wavelength peaks are resonance peaks. At shorter wavelengths, the first series of thickness peaks and at even shorter wavelengths, the second series of thickness peaks (see also Fig. 3). 


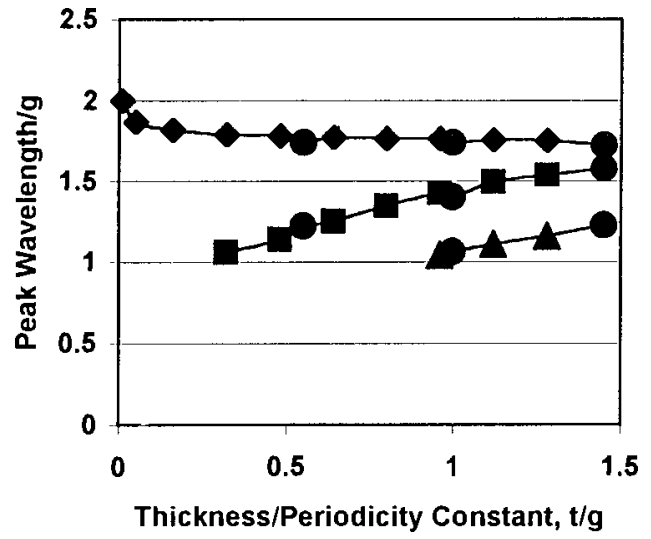

FIG. 3. Graph of wavelengths of resonance and thickness peaks divided by the periodicity constant $g=20 \mu \mathrm{m}$, plotted depending on $(t / g)$, the ratio of metal thickness to periodicity constant. Upper curve: Resonance wavelengths. Diamonds calculated with the Micro-Stripes program. Round dots are experimental data. Next lower curve: Thickness peaks. Squares calculated with the Micro-Stripes program. Round dots are experimental data. Lowest curve: Thickness peaks. Triangles calculated with the Micro-Stripes program. Round dots are experimental data.

\section{B. The oscillator model and transmission line theory}

\section{Oscillator model}

Ulrich ${ }^{13}$ has analyzed the modes of thin square shaped inductive metal meshes. The modes are composed of pairs of surface waves tightly bound by the openings. The corresponding wavelengths are the resonance wavelengths of a thin metal mesh. We extended this model to thick metal meshes and assumed that each of the surface waves on the front and back side of the thick mesh are described by an oscillator. The oscillators are coupled by waveguide modes of the openings, depending on the thickness $t$ of the mesh. For a qualitative description of this simple model of two interacting oscillators we use transmission line theory ${ }^{14}$ and calculate the peaks of the transmitted waves.

\section{Transmission line theory}

Ulrich developed the transmission line theory for the calculation of reflectance and transmittance of thin metal meshes. ${ }^{5}$ The theory uses geometrical and electrical parameters for the description of optical properties: a short description is given in Ref. 15. Ulrich's formulation has been corrected by Whitbourn and Compton ${ }^{16}$ and the revised formulation will be used here.

a. Oscillator. We considered an inductive thin metal mesh and described the resonance oscillation by a shunt impedance (see Fig. 6),

$$
Y(\lambda)=1 /\left[a 1-i\left(\omega_{0} A 1\right) / \Omega(\lambda)\right],
$$

where

$$
\Omega(\lambda)=\left(g / \lambda \omega_{0}\right)-\left(\lambda \omega_{0} / g\right) .
$$

The periodicity constant of the mesh is $g$, the resonance frequency $\omega_{0}$, the bandwidth parameter $A 1$ and the loss parameter $a 1$. The input parameters $\omega_{0}, A 1$, and $a 1$ may be obtained from observations or simulations such as the MicroStripes program or empirical formulas (see Sec. II B 3).

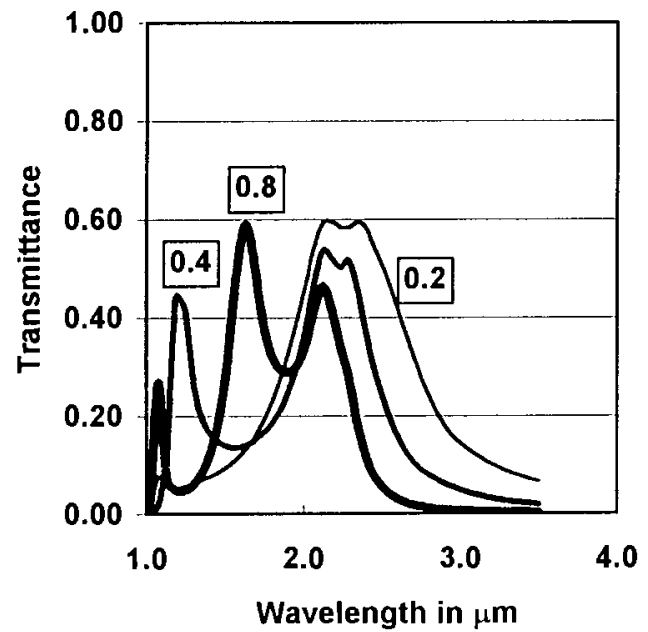

(a)

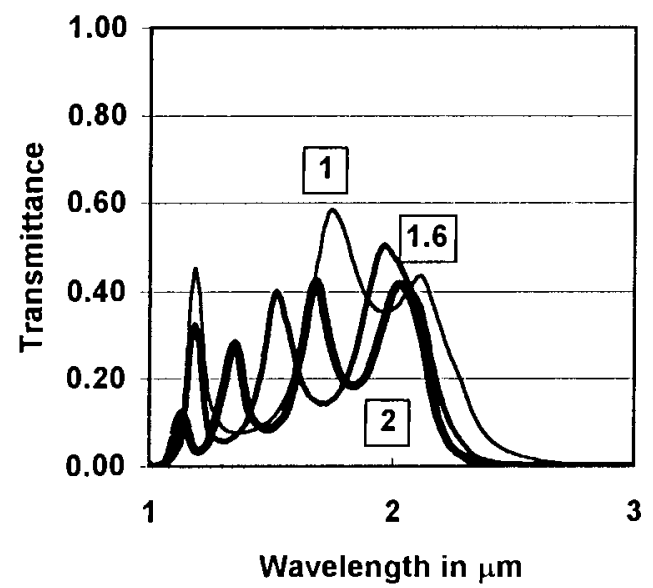

(b)

FIG. 4. (a) Transmittance of inductive cross shaped meshes calculated with the Fourier modal method. Geometrical parameters $g=1 \mu \mathrm{m}, 2 a$ $=0.2 \mu \mathrm{m}$, and $2 b=0.11 \mu \mathrm{m}$. The metal thicknesses $0.2,0.4$, and $0.8 \mu \mathrm{m}$ are indicated in the graph area. The long wavelength peaks are resonance peaks, the short wavelength peaks are the first series of thickness peaks (see also Fig. 5). (b) Transmittance of inductive cross shaped meshes calculated with the Fourier modal method. Geometrical parameters $g=1 \mu \mathrm{m}, 2 a$ $=0.2 \mu \mathrm{m}$, and $2 b=0.11 \mu \mathrm{m}$. The metal thicknesses $1,1.6$, and $2 \mu \mathrm{m}$ are indicated in the graph area. The longest wavelength peaks are resonance peaks. At shorter wavelengths the first series of thickness peaks, at even shorter wavelengths, the second series of thickness peaks (see also Fig. 5).

b. Cascading matrices. It is convenient to use the cascading matrix formulation (see Timusk and Richards ${ }^{17}$ ). Each element of the transmission line is presented by a $2 \times 2$ matrix. The waves on the left side of the impedance $Y$ (see Fig. 6), are related by a matrix $M$ to the waves on the right side as

$$
\begin{aligned}
& b_{1}=m_{11} a_{2}+m_{12} b_{2}, \\
& a_{1}=m_{21} a_{2}+m_{22} b_{2} .
\end{aligned}
$$

For $a_{2}=0$ (that is, no "backwards traveling wave" on the right side), the ratio of the reflected wave $b_{1}$ to incident wave $a_{1}$ is

$$
b_{1} / a_{1}=m_{12} / m_{22},
$$




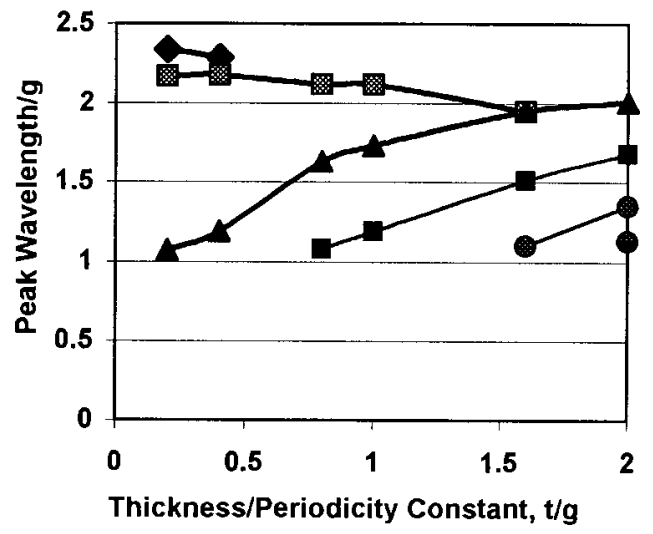

FIG. 5. Graph of wavelengths of resonance and thickness peaks divided by the periodicity constant $g=1 \mu \mathrm{m}$, plotted depending on $(t / g)$, the ratio of metal thickness to periodicity constant. Upper curves: Squares and diamonds are resonance wavelengths. Next lower curve: Triangles are the first series of thickness peaks. Two lowest curves: Second and third series of thickness peaks.

and the ratio of the transmitted wave $b_{2}$ to the incident wave $a_{1}$ is

$$
b_{2} / a_{1}=1 / m_{22} \text {. }
$$

The transmitted intensity is calculated from the $(2,2)$ element of the resulting product matrix, presenting all elements in the transmission line.

The matrix $M 1$ of the impedance $Y$ has the elements

$$
\begin{aligned}
& m 1_{11}=(-Y / 2+1), \quad m 1_{12}=-Y / 2, \\
& m 1_{21}=Y / 2, \quad m 1_{22}=(Y / 2+1),
\end{aligned}
$$

and the matrix $M 2$ of the separation of length $t$ in the medium with refractive index 1

$$
\begin{aligned}
& m 2_{11}=\exp (-i 2 \pi t) / \lambda, \quad m 2_{12}=0, \\
& m 2_{21}=0, \quad m 2_{22}=\exp (i 2 \pi t) / \lambda .
\end{aligned}
$$

To include losses one may take the refractive indices as a complex quantity.

\section{Calculations}

The two oscillators of our model are described by the matrix product $M=M 1 M 2 M 1$, where the separation of the oscillators corresponds to the thickness $t$ of the metal mesh. The input data are as follows: The resonance frequency $\omega_{o}$ is obtained from $g / \lambda_{R}$, where $\lambda_{R}$ is taken from the empirical formula $\lambda_{R}=2 g-4 a+2 b$ of the resonance wavelength of thin inductive metal meshes, ${ }^{6}$ in agreement with

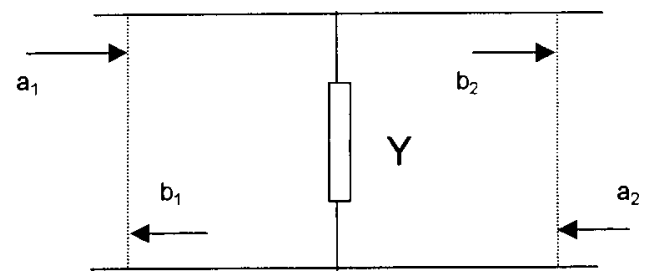

FIG. 6. Transmission line presentation. Shunt impedance with incident and reflected waves on the left side and transmitted and backward traveling waves on the right side.

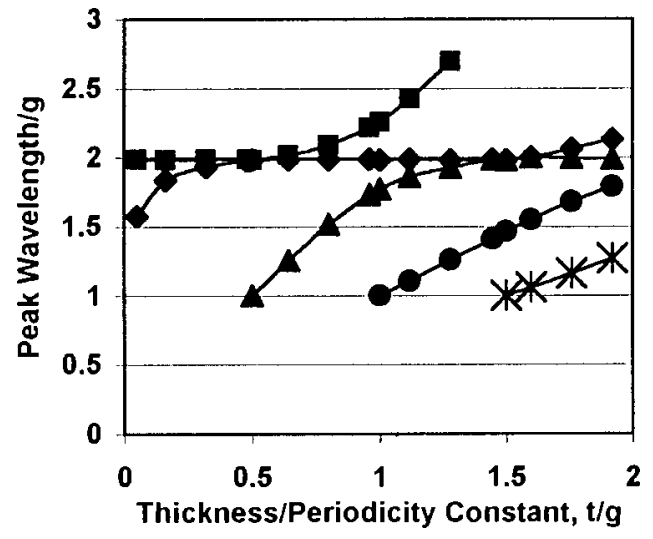

FIG. 7. Transmission line theory calculations of resonance and thickness peaks of two oscillators at distance $t$. The peak wavelength divided by the periodicity constant $(t / g)$ of resonance and thickness peaks are plotted depending on $(t / g)$, the ratio of metal thickness to periodicity constant. At small values of $t / g$, the resonance peaks show interaction and have $\lambda / g$ values around 2 . At $t / g=0.5$, the interaction of the resonance peaks has vanished and the first branch of thickness peaks appears. At $t / g=1$, the first peak of the second series of thickness peaks appears. At $t / g=1.5$, the first series of thickness peaks interacts strongly with the resonance peaks.

Micro-Stripes ${ }^{11}$ calculations. The band width parameter, obtained from Micro-Stripes calculations, is $A 1=0.1$, and the loss parameter $a 1$ is 0.001 , according to Ref. 16 .

In Fig. 7 we have plotted $(\lambda / g)$, the calculated peak wavelengths divided by the periodicity constant, versus $(t / g)$, the thickness divided by the periodicity constant, for the range of $t / g$ from 0 to 2 .

For small $t / g$ values, the two oscillators interact and show a "splitting," which decreases for increasing values of $t / g$. At around $t / g=0.6$ a new peak appears, shifting for larger values of $t / g$ towards the wavelength of the oscillators. At around $t / g=1$, the wavelength of the "new peak" and the peaks of the oscillators are close together and interact. The interaction of the oscillators with one another and the "new peaks" is small and therefore the resulting wavelength is close to the resonance wavelength of the thin mesh. The "new peaks" are the "thickness peaks" and are interpreted as compound modes composed of oscillator resonance and waveguide modes. ${ }^{10}$ The waveguide modes are "FabryPerot" modes, as they appear between two reflecting plates, similar to those described in Ref. 18 for narrow slots in thick gratings. The second and third series are also "thickness peaks," composed of resonance and higher order waveguide modes. All three branches of "thickness peaks" appear with their first peak at a wavelength about equal to the periodicity constant $g$. Interaction occurs when the thickness $t$ is in the range of the periodicity constant.

\section{DISCUSSION}

The oscillator model describes the transmittance of the mesh by the interaction of the oscillator resonance modes and the waveguide mode. The incident light at normal incidence induces a standing wave mode within the mesh. The mode couples energy of the incident light to the transmitted and reflected lights. This induced resonance mode is composed of two standing surface waves, one on each side of the 
mesh. When the surface waves interact through the openings, degeneracy is lifted and the resonance is split into two. As the metal thickness increases, the coupling decreases and the resonance peaks are merged into one peak. This is shown in simulations for the short wavelength region as a splitting disappearing for large thickness [Fig. 4(a)]. In the long wavelength region, simulations and experiments show a broad peak decreasing in width [Fig. 2(a)]. With increasing metal structure thickness, the interaction of the two surface waves decreases and the energy transfer is now accomplished by wave guide modes. The simulations in both spectral regions and the experiments in the long wavelength region show several series of new peaks with peak wavelengths depending on the thickness of the mesh and shifting to longer wavelengths.

In this article we have applied the oscillator model to interpret transmittance data of cross shaped meshes. The geometrical property of crosses is contained in the input data of the oscillators used in transmission line theory. These input data will be different for square or hole apertures, but the qualitative result will not significantly change. MartinMoreno et al. ${ }^{10}$ have calculated thick periodic structures with square shaped openings in the short wavelength region. Transmission data are reported for free-standing meshes with periodicity constant $g=750 \mathrm{~nm}$, square length of $280 \mathrm{~nm}$, and five thicknesses with $t / g$ values of $0.13,0.26,0.4,0.53$, and 0.67. The transmittances, depending on thicknesses of these meshes, show a resonance and a thickness peak, very similar to our results for crosses, as expected from the oscillator model. A thickness peak appears first for $t / g=0.13$ at a wavelength close to $g$. For increasing thickness, the thickness peaks shift to longer wavelength while the resonance peak shifts to shorter wavelength and both appear together in the range of $t / g=0.4-0.67$.

The results of Ref. 10 have been applied to the interpretation of experimental data on round holes, studied by Ebbesen $e$ t $a l^{8}{ }^{8}$ on substrates, and by Grupp et al. ${ }^{9}$ as free standing structures. Unfortunately, these experimental investigations are done only for a narrow range of $t / g$ values. In Ref. 9, Fig. 2, one peak is shown, perhaps with an additional shoulder, at $t / g$ of about 0.4 for round holes of diameter $400 \mathrm{~nm}$ and $g=750 \mathrm{~nm}$. For the same $t / g$ values and the same $g$, at shorter wavelengths (Ref. 10), though, a broad peak with a dip is exhibited for squares of length of $280 \mathrm{~nm}$.

In our opinion, there is no extraordinary transmission enhancement in the short wavelength region. In order to normalize the transmittance properly, one needs to take the ratio of the area of the aperture to the unit cell area. The percentage transmittance of the experimental data in the short and long wavelength regions is about 2 to 3 times larger than this ratio. These transmittance enhancements occur via resonance modes, as mentioned for the short wavelength region in Ref. 10 and follows from Ref. 13 for the long wavelength region. We consider it an open question how to compare the transmittance of one hole with a periodic structure.

\section{SUMMARY}

The experimental and simulated transmittance spectra of thick cross-shaped metal meshes have been studied in the short and long wavelength regions. These spectra exhibit transmission peaks with rather similar spectral appearance depending on the mesh thickness. A simple oscillator model reproduces very well the peak wavelengths depending on thickness. This qualitative model supports the interpretation of the peaks as resonances of the surface waves and "FabryPerot" modes of the openings, coupled to the surface waves on both sides of the mesh. The interpretation using the oscillator model applies very well to arrays of crosses, squares, or holes in the short and long wavelength region.

\section{ACKNOWLEDGMENT}

This work was supported by the National Science Foundation, Grant No. ECS-9820200.

${ }^{1}$ T. W. Ebbesen, H. J. Lezec, H. F. Ghaemi, T. Thio, and P. A. Wolff, Nature (London) 391, 667 (1998).

${ }^{2}$ D. E. Grupp, H. J. Lezec, T. W. Ebbesen, K. M. Pellerin, and T. Thio, Appl. Phys. Lett. 77, 1569 (2000).

${ }^{3}$ L. Martin-Moreno, F. J. Garcia-Vidal, H. J. Lezec, K. M. Pellerin, T. Thio, J. B. Pendry, and T. W. Ebbesen, Phys. Rev. Lett. 86, 1114 (2001).

${ }^{4}$ R. Ulrich, K. F. Renk, and L. Genzel, IEEE Trans. Microwave Theory Tech. 11, 363 (1963).

${ }^{5}$ R. Ulrich, Infrared Phys. 7, 37 (1967).

${ }^{6}$ S. T. Chase and R. D. Joseph, Appl. Opt. 22, 1774 (1983).

${ }^{7}$ D. M. Byrne, Proc., SPIE 560, 70 (1985).

${ }^{8}$ R. Ruprecht, W. Bacher, P. Bley, M. Harmening, and W. K. Schomburg, KfK-Nachr. Jahrg. 23, 2-91 (1991); 23, 18-123 (1991).

${ }^{9}$ C. Compton, R. D. McPhedran, G. H. Derrick, and L. C. Botten, Infrared Phys. 23, 239 (1983).

${ }^{10}$ K. D. Möller, K. R. Farmer, D. V. P. Ivanov, O. Sternberg, K. P. Stewart, and P. Lalanne, Infrared Phys. 40, 475 (1999).

${ }^{11}$ Micro-Stripes Program by Flomerics, Inc., 275 Turnpike Road, Suite 100, Southborough, MA 01772.

${ }^{12}$ L. Li, J. Opt. Soc. Am. A 14, 2758 (1997).

${ }^{13} \mathrm{R}$. Ulrich, Proceedings of the Symposium of Optical and Acoustical Micro-Electronics, New York, NY, 1974 (Polytechnic, Brooklyn, NY, 1974), Vol. XXIII.

${ }^{14}$ D. M. Kearns and R. W. Beatty, Basic Theory of Waveguide Functions and Introductory Microwave Network Analysis (Pergamon, Oxford, 1967).

${ }^{15}$ K. D. Möller and W. G. Rothschild, Far Infrared Spectroscopy (Wiley, New York, 1971).

${ }^{16}$ L. B. Whitbourn and R. C. Compton, Appl. Opt. 24, 217 (1985).

${ }^{17}$ T. Timusk and P. L. Richards, Appl. Opt. 20, 1355 (1981).

${ }^{18} \mathrm{~S}$. Astilean, Ph. Lalanne, and M. Palamaru, Opt. Commun. 175, 256 (2000). 